\title{
CATEGORIES OF MOTIVES FOR ADDITIVE CATEGORIES. I
}

\author{
A. V. YAKOVLEV \\ Dedicated to the centenary of the birth of Dmitriu Konstantinovich Faddeev
}

\begin{abstract}
In line with the development of a theme of a classical paper by D. K. Faddeev, motivic extensions of certain additive categories are studied.
\end{abstract}

\section{§1. INTRODUCTION}

The paper [1] is one of the most frequently cited papers of D. K. Faddeev. Usually, the content of that paper is not touched upon; it is only stated that, together with lectures given by D. K. Faddeev at several conferences, that paper was one of the impulses for the subsequent rapid development of the theory of integral representations. However, there are plenty of very interesting ideas and results in 1, which are not yet quite understood by mathematicians. My paper 2] was in great measure inspired by these ideas. In 1], an approach to the study of representations of finitely generated modules over Dedekind rings was developed. But I was interested in torsion free Abelian groups of finite rank; in general, they are not generated by any finite set of elements, and for this reason I could not apply directly the results of Dmitriu Konstantinovich. However, using his ideas, I succeeded in developing a fairly substantial theory of the objects that interested me; in particular, this theory completely explains the nonuniqueness of direct decompositions of such groups.

One of the ideas going back to [1] consisted in the extension of the base ring. For example, one can consider the category whose objects are again torsion free Abelian groups of finite rank, but the homomorphism groups are obtained from the usual homomorphism groups by tensor multiplication by a certain ring (for example, the field of rational or complex numbers, or the ring of $p$-adic integers). However, it may happen that the endomorphism ring of an Abelian group in the new category contains idempotent endomorphisms that do not correspond to any direct summand of the group. Therefore, it becomes necessary to adjoin new objects to the category; these objects were called "imaginary direct summands" in [2]. Later, together with my postgraduate student N'Famara Kamara, we developed in 3 a similar theory for mixed Abelian groups satisfying some finite rank conditions.

The idea of adjoining some nonexisting objects is not new; it appears in other branches of mathematics as well. For example, the so-called "Grothendieck motives" have long been studied in algebraic geometry; these motives are none other than adjoined objects that correspond to idempotent endomorphisms of an additive category consisting of algebraic varieties. Therefore, it seems natural to construct a general theory applicable in various situations.

2000 Mathematics Subject Classification. Primary 13C99, 18E05.

Key words and phrases. Additive category, category of motives, genus, almost indecomposable object. 
The results of the papers [2, 3] are fairly meaningful, and the proofs are based on the study of endomorphism rings. Therefore it seemed that it should not be difficult to extend these results to arbitrary additive categories, the objects of which satisfy some finiteness conditions. However, it turned out that it was not so easy to find the restrictions on the category under which the results of [2, 3] remain valid; my purpose in this paper is the description of such categories.

The results we want to obtain can be described as follows. Throughout the paper, $\mathcal{P}$ is the set of all prime integers and $\mathcal{P}_{0}=\mathcal{P} \cup\{0\}$.

Let $\mathfrak{A}$ be an additive category; we embed it in the category of motives $M=M(\mathfrak{A})$, adjoining all "imaginary direct summands" to it. Assume that the category $\mathfrak{A}$ satisfies conditions $(*),(* *)$, and $(* * *)$ stated in $\S 2$, and that the category of motives $M$ satisfies conditions (i) and (ii) in $\S 3$. We construct categories $M_{l}(l \in \mathcal{P}), M_{0}, M_{\infty}$ and the functors shown in the commutative diagram

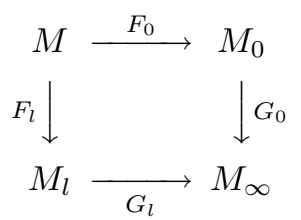

(this has turned out to be more difficult than in the situation considered in 2]). The homomorphism groups in the categories $M_{0}$ and $M_{\infty}$ are finite-dimensional vector spaces over the fields of rational and complex numbers, and the homomorphism groups in the category $M_{l}$ may have torsion that is a finite $l$-group, but the factor groups of the homomorphism groups over torsion subgroups must be torsion free modules of finite rank over the ring of $l$-adic integers.

We need some more definitions. We say that two objects $A, B$ of the category $M$ belong to the same genus if the objects $F_{0}(A), F_{0}(B)$ of the category $M_{0}$ are isomorphic and the objects $F_{l}(A), F_{l}(B)$ of the categories $M_{l}$ are isomorphic for all primes $l$. An object $A$ of the category $M$ is said to be almost indecomposable if for any direct decomposition $A=X \oplus Y$ of this object one of the objects $F_{0}(X), F_{0}(Y)$ is the zero object of the category $M_{0}$.

The following theorem is true for categories $\mathfrak{A}$ and $M$ that satisfy the above conditions.

Theorem 1. The Krull-Schmidt theorem holds in the categories $M_{0}, M_{l}, M_{\infty}$ (but not in $M !)$.

2. If $A, B$ are objects of the category $M$ that belong to the same genus, and $A=X \oplus Y$, then there exists a direct decomposition $B=X^{\prime} \oplus Y^{\prime}$ such that $X^{\prime}$ and $X$ belong to the same genus, and $Y^{\prime}$ and $Y$ belong to the same genus.

3. If $A, B$ are objects of the category $M$ that belong to the same genus, and the object $A$ is almost indecomposable, then the object $B$ is also almost indecomposable.

4. For each $l \in \mathcal{P}_{0}$, choose an object $X_{l}$ of the category $M_{l}$. If the objects $G_{l}\left(X_{l}\right)$ and $G_{0}\left(X_{0}\right)$ of the category $M_{\infty}$ are isomorphic for all primes $l$, and almost all objects $X_{l}$ are trivial in a certain sense (which we shall define later), then there exists an object $A$ of the category $M$ such that $F_{l}(A) \approx X_{l}$ for all $l \in \mathcal{P}_{0}$. It is clear that any two such objects belong to the same genus.

In this paper we realize only a part of this program. Namely, we describe the class of categories for which we shall later prove Theorem 1 , and we construct a new category $\bar{M}$ that, as we shall show, is a good approximation to the category $M$. The construction of the categories $M_{l}, M_{\infty}$ and the proof of Theorem 1 will be given in a forthcoming paper. 


\section{$\S 2$. Category $\mathfrak{A}$}

Let $\mathfrak{A}$ be an additive category. For any objects $A, B$ of $\mathfrak{A}$ we shall use the following notation.

- $H(A, B)=\operatorname{Hom}_{\mathcal{A}}(A, B)$;

- $T(A, B)$ is the group of periodic elements of the additive group $H(A, B)$;

- $T_{l}(A, B)(l \in \mathcal{P})$ is the $l$-component of the group $T(A, B)$;

- $D_{0}(A, B)$ is the set of all divisible elements of the group $H(A, B), 1$

- $D(A, B)$ is the set of all elements $a \in H(A, B)$ such that for each integer $n \neq 0$ there exist elements $x \in T(A, B)$ and $b \in H(A, B)$ for which $a=n b+x$.

It is obvious that $D(A, B) \supseteq T(A, B)+D_{0}(A, B)$ and that $D(A, B) / T(A, B)$ is the group of all divisible elements of the group $H(A, B) / T(A, B)$. Moreover, the groups $D_{0}(A, B)$ and $H(A, B) / D(A, B)$ are torsion free.

We assume that the category $\mathfrak{A}$ satisfies the following "finiteness" condition for its objects:

(*) For any objects $A, B$ of the category $\mathfrak{A}$, all groups $T_{l}(A, B)$ are finite $(l \in \mathcal{P})$, and the groups $D_{0}(A, B), H(A, B) / D(A, B)$ are torsion free Abelian groups of finite rank.

For an object $A$ of $\mathfrak{A}$, we denote its endomorphism ring by $E(A)$, the group of periodic elements of the additive group of the $\operatorname{ring} E(A)$ by $T(A)$, and the $l$-component of the group $T(A)$ by $T_{l}(A)(l \in \mathcal{P})$. Clearly, $T(A)$ and $T_{l}(A)$ are two-sided ideals of the ring $E(A)$, and our assumption implies that all rings $T_{l}(A)$ are finite.

Lemma 1. For every prime $l$, there is an idempotent element $e_{l}(A) \in T_{l}(A)$ such that for each $x \in T_{l}(A)$ the element $x-e_{l}(A) x$ is contained in the nilpotent radical of the ring $E(A)$.

Proof. For simplicity, we write $E, T_{l}$, and $e_{l}$ instead of $E(A), T_{l}(A)$, and $e_{l}(A)$. Let $N_{l}$ be the nilpotent radical of the ring $T_{l}$. Since the $\operatorname{ring} T_{l}(A)$ is finite, the ideal $N_{l}$ is nilpotent; consequently, it coincides with the Jacobson radical of the $\operatorname{ring} T_{l}$. Therefore, either the factor ring $T_{l} / N_{l}$ is zero (and then we can take $e_{l}=0$ ), or it is a nonzero semisimple ring, and then it is a ring with unity $\bar{e}$. We can lift the idempotent $\bar{e}$ modulo the nilpotent ideal $N_{l}$ up to an idempotent element $e_{l} \in T_{l}$. Since the class of $e_{l}$ modulo $N_{l}$ is the unity of the factor ring $T_{l} / N_{l}$, we see that $x-e_{l} x \in N_{l}$ for any $x \in T_{l}$. It remains to observe that the ideal of the $\operatorname{ring} E$ generated by $N_{l}$ is a nilpotent ideal. Indeed, if $N_{l}^{n}=0$, then

$$
\left(E N_{l} E\right)^{2 n}=\left(E N_{l} E N_{l}\right)^{n} \subseteq\left(E T_{l} E N_{l}\right)^{n} \subseteq\left(T_{l} N_{l}\right)^{n} \subseteq N_{l}^{n}=0 .
$$

In this paper we restrict ourselves to the study of categories in which the idempotents $e_{l}(A)$ constructed above satisfy a stronger condition: $x-e_{l}(A) x=0$ for all $x \in T_{l}(A)$. In other words, the element $e_{l}(A)$ is a left unity of the $\operatorname{ring} T_{l}(A)$ (but not necessarily a right unity). This restriction is not really important: this can be ensured by passing to the factor ring of $E(A)$ over an ideal contained in the nilpotent radical of the ring $E(A)$, and in all respects that are of interest for us the factor ring modulo the nilpotent radical is similar to the ring itself, but without this restriction the statements become much more cumbersome. We shall give more details in our forthcoming publications. Thus, we assume that the category $\mathcal{A}$ satisfies the following condition:

(**) for any object $A$ of the category $\mathfrak{A}$, and any prime integer $l$, the ring $T_{l}(A)$ contains a left unity $e_{l}(A)$.

\footnotetext{
${ }^{1}$ Recall that an element of an Abelian group is said to be divisible if it is divisible by all nonzero integers.
} 
Furthermore, we assume that

(***) for any objects $A, B$ of the category $\mathfrak{A}$, the group $\operatorname{Hom}_{\mathcal{A}}(A, B)$ contains no nonzero divisible element.

Like $(* *)$, this restriction is not really important, and it can easily be lifted (though the formulations become a little more complicated). Much stronger restrictions (though looking innocent) will be imposed in the following sections.

\section{§3. Category of motives For the CAtegory $\mathfrak{A}$}

It may happen that an idempotent endomorphism of an object of the category $\mathfrak{A}$ does not correspond to any direct summand of this object. We extend the category by adjoining new objects to it; in [2] the author named these new objects "imaginary" direct summands. Namely, the objects of the extended category $M(\mathfrak{A})$ are pairs $(A, e)$ consisting of an object $A$ of $\mathfrak{A}$ and an idempotent element $e$ of the endomorphism ring $E(A)$ of the object $A$; the homomorphism groups in $M(\mathfrak{A})$ are defined by the formula

$$
\operatorname{Hom}_{M(\mathfrak{A})}((A, e),(B, d))=d\left(\operatorname{Hom}_{\mathfrak{A}}(A, B)\right) e \subseteq \operatorname{Hom}_{\mathfrak{A}}(A, B) .
$$

It is natural to call this new category the category of motives for the additive category $\mathfrak{A}$.

In what follows we shall ignore the category $\mathfrak{A}$ and almost exclusively study its category of motives $M(\mathfrak{A})$, denoting the latter simply by $M$. Obviously, $M$ is an additive category satisfying conditions $(*),(* *),(* * *)$; moreover, every idempotent endomorphism of an object of the category $M$ corresponds to a direct summand of this object.

Let $l$ be a prime integer; we say that an object $A$ of $M$ is l-periodic if $E(A)=T_{l}(A)$. Though the additivity of a category only implies the existence of finite direct sums, we shall assume that, in a very special situation, certain infinite direct sums exist in $M$.

(i) For each prime integer $l \in \mathcal{P}$, let $A_{l}$ be an l-periodic object of the category $M$ (some of the $A_{l}$ can be zero objects). Then the direct sum of these objects $\bigoplus_{l \in \mathcal{P}} A_{l}$ exists in the category $M$.

Certainly, we could formulate condition (i) in terms of the category $\mathfrak{A}$ itself, rather than in terms of its category of motives $M=M(\mathfrak{A})$; but then the condition would not be so transparent. Later on, for an arbitrary object $A$ of the category $M$ we shall construct its torsion $t(A)$; the torsion of $A$ is a subobject of $A$, and it is an infinite direct sum of $l$-periodic objects.

Let $A_{l}(l \in \mathcal{P})$ and $B$ be objects of $M$ such that $A_{l}$ is an $l$-periodic object for each $l$. Next, let $a_{l}: A_{l} \rightarrow B$ be homomorphisms given for all $l \in \mathcal{P}$. Then, by the definition of the direct sum, there exists a unique homomorphism

$$
a=\sum_{l \in \mathcal{P}} a_{l}: \bigoplus_{l \in \mathcal{P}} A_{l} \rightarrow B
$$

the compositions of which with the canonical embeddings $A_{l} \rightarrow \bigoplus_{l \in \mathcal{P}} A_{l}$ coincide with the homomorphisms $a_{l}$. It will be helpful for us to require that, whenever infinite direct sums appear, the direct sum of monomorphisms be a monomorphism as well.

(ii) Let $A_{l}(l \in \mathcal{P})$ and $B$ be objects of the category $M$ such that the objects $A_{l}$ are l-periodic for all $l$, and let $a_{l}: A_{l} \rightarrow B$ be monomorphisms. Then the homomorphism

$$
\sum_{l \in \mathcal{P}} a_{l}: \bigoplus_{l \in \mathcal{P}} A_{l} \rightarrow B
$$

is also a monomorphism.

Again, let $A_{l}(l \in \mathcal{P})$ be $l$-periodic objects of the category $M$. We put $A=\bigoplus_{l \in \mathcal{P}} A_{l}$ and let $u_{l}$ be endomorphisms of the objects $A_{l}$. Let $u_{l}^{\prime}$ denote the composition of $u_{l}$ with the canonical embedding of the direct summand $A_{l}$ into $A$. By the definition of the 
direct sum, there exists a unique homomorphism $u$ of $A=\bigoplus_{l \in \mathcal{P}} A_{l}$ to $A$ such that for each $l \in \mathcal{P}$ the composition of the canonical embedding $A_{l} \rightarrow A$ with $u$ is equal to $u_{l}^{\prime}$. This endomorphism of the object $A$ will be denoted by

$$
\prod_{l \in \mathcal{P}} u_{l}: \bigoplus_{l \in \mathcal{P}} A_{l} \rightarrow \bigoplus_{l \in \mathcal{P}} A_{l}
$$

\section{§4. The TORSION OF OBJECTS OF $M$}

Until the end of $\S 5$, we fix an object $A$ of $M$; for brevity we shall write $E, T, T_{l}, e_{l}$ instead of $E(A), T(A), T_{l}(A), e_{l}(A)$.

The element $e_{l} \in T_{l} \subseteq E$ is an idempotent endomorphism of the object $A$. Hence, by the definition of the category $M$, we have the corresponding direct decomposition $A=A_{l}^{\prime} \oplus A_{l}$. We denote by $i_{l}: A_{l} \rightarrow A$ and $p_{l}: A \rightarrow A_{l}$ the canonical embedding of the summand $A_{l}$ in the direct sum $A=A^{\prime} \oplus A_{l}$ and the canonical projection of this direct sum onto $A_{l}$. We shall call the infinite direct sum $\bigoplus_{l \in \mathcal{P}} A_{l}$ the periodic part or (torsion) of the object $A$ and denote it by $t(A)$; the inclusions $i_{l}$ induce the homomorphism $i=i^{A}: t(A)=\bigoplus_{l \in \mathcal{P}} A_{l} \rightarrow A$. Since the canonical embeddings $i_{l}$ of the objects $A_{l}$ into the direct sums $A=A_{l} \oplus A_{l}^{\prime}$ are monomorphisms, the homomorphism $i^{A}=\sum_{l \in \mathcal{P}} i_{l}$ is also a monomorphism (by condition (ii)). Thus, the torsion $t(A)$ is a subobject of $A$.

Lemma 2. For any prime integer $l$, the following relations are true: $i p_{l}=i_{l} p_{l}=e_{l}$, $p_{l} i_{l}=\mathrm{id}_{A_{l}}, e_{l} i_{l}=i_{l}, p_{l} e_{l}=p_{l}$. If $l, q$ are distinct prime integers, then $p_{l} i_{q}=0$ and consequently $e_{l} i_{q}=0, e_{l} e_{q}=0, p_{l} e_{q}=0$.

Proof. The relations $i_{l} p_{l}=e_{l}, p_{l} i_{l}=\mathrm{id}_{A_{l}}$ follow immediately from the definitions and imply the relations $e_{l} i_{l}=i_{l}, p_{l} e_{l}=p_{l}$. We only need to prove that $p_{l} i_{q}=0$ if $l \neq q$. But this is also trivial: $l e_{l}=q e_{q}=0$ because $e_{l} \in T_{l}, e_{q} \in T_{q}$. Therefore,

$$
l p_{l} i_{q}=l p_{l} e_{l} i_{q}=0, \quad q p_{l} i_{q}=q p_{l} i_{q} e_{q}=0 .
$$

Since $l$ and $q$ are relatively prime, we obtain $p_{l} i_{q}=0$.

Now we show that each endomorphism of the object $A$ maps $t(A)$ into itself. More precisely, if $a \in E$, then there exists an endomorphism $u$ of the object $t(A)$ such that $a i=i u$.

Lemma 3. For any $b \in E$ and any prime integer $l$ we have $b i_{l}=i_{l} p_{l} b i_{l}$.

Proof. The element $b e_{l}$ is contained in $T_{l}$ because $e_{l} \in T_{l}$ and $T_{l}$ is an ideal of $E$; therefore, $b e_{l}=e_{l} b e_{l}$. By Lemma 2 we obtain $b i_{l}=b e_{l} i_{l}=e_{l} b e_{l} i_{l}=i_{l} p_{l} b i_{l}$.

Lemma 4. For any $b \in E$ we have $b i=i \prod_{l \in \mathcal{P}} p_{l} b i_{l}$.

Proof. Since both sides of the formula are homomorphisms from the direct sum $t(A)=$ $\bigoplus A_{l}$ to $A$, it suffices to check that their restrictions to the direct summands coincide. But the restriction of the left-hand side to $A_{l}$ is equal to the homomorphism $b i_{l}$, which is equal to $i_{l} p_{l} b i_{l}=i p_{l} b i_{l}$ by Lemmas 2 and 3 , the restriction of the right-hand side to $A_{l}$ is obviously the same.

Lemma 44 shows that any endomorphism of the object $A$ maps the subobject $t(A)$ into itself: if $b \in E$, then there exists an endomorphism $u$ of the object $t(A)$ such that $b i=i u$. Thus, the object $t(A)$ meets the usual requirements on torsion: it is a fully characteristic subobject of $A$. 


\section{§5. ENDOMORPHISMS THAT FACTOR THROUGH THE TORSION}

We say that a homomorphism $a: B \rightarrow A$ factors through the periodic part $t(A)$ of $A$ if there exists a homomorphism $a^{\prime}: B \rightarrow t(A)$ such that $a=i a^{\prime}$ (recall that $i$ stands for the canonical embedding of the periodic part $t(A)$ of $A$ into $A$ ).

Lemma 5. If a homomorphism $a: B \rightarrow A$ factors through the periodic part $t(A)$ of the object $A$, then the coset of a modulo $T(B, A)$ is a divisible element of the factor group $H(B, A) / T(B, A)$.

Proof. By assumption, there is a homomorphism $a^{\prime}: B \rightarrow t(A)=\bigoplus_{l \in \mathcal{P}} A_{l}$ such that $a=i a^{\prime}$. For each prime integer $l$, let $a_{l}^{\prime}$ denote the composition of $a^{\prime}$ and the canonical projection of the direct sum onto $A_{l}$; we set $a_{l}=i_{l} a^{\prime} l$. It is clear that $a_{l} \in T_{l}(B, A) \subseteq$ $T(B, A)$ and that $a-a_{l}: B \rightarrow A$ factors through the direct sum $\bigoplus_{q \neq l} A_{q}$, in which the division by any power of $l$ is possible. Therefore, the coset of $a$ modulo $T(B, A)$ is divisible by any power of $l$. Since $l$ is an arbitrary prime, this coset is divisible by any nonzero integer.

We say that an endomorphism $a \in E$ of the object $A$ is weakly nilpotent if there exists an integer $n>0$ such that the homomorphism $a^{n}$ factors through the periodic part $t(A)$ of $A$, and moreover, for each prime $l$ there exists an integer $n_{l}>0$ such that the restriction $p_{l} a^{n_{l}} i_{l}$ of $a^{n_{l}}$ to $A_{l}$ is the zero homomorphism. If the numbers $n_{l}$ are uniformly bounded, then a weakly nilpotent endomorphism $a$ is nilpotent; but this is not always the case, and a weakly nilpotent endomorphism may fail to be nilpotent.

Lemma 6. Let $a^{\prime}$ be a homomorphism from $A$ to $t(A)$, let $a=i a^{\prime}$, and let $b \in E$ be an endomorphism of $A$ such that $b a=a b$. For all prime $l$ choose integers $s_{l} \geq 1$ and set $c=i\left(\prod p_{l} a^{s_{l}} i_{l}\right) a^{\prime}$. Then $b c=c b$.

Proof. The relation $b a=a b$ and Lemma 4 imply that

$$
i a^{\prime} b=a b=b a=b i a^{\prime}=i\left(\prod_{l \in \mathcal{P}} p_{l} b i_{l}\right) a^{\prime}
$$

Since $i$ is a monomorphism, we obtain $a^{\prime} b=\left(\prod_{l \in \mathcal{P}} p_{l} b i_{l}\right) a^{\prime}$. Next, the homomorphisms

$$
i\left(\prod_{q \in \mathcal{P}} p_{q} b i_{q}\right)\left(\prod_{l \in \mathcal{P}} p_{l} a^{s_{l}} i_{l}\right),\left(i \prod_{l \in \mathcal{P}} p_{l} a^{s_{l}} i_{l}\right)\left(\prod_{q \in \mathcal{P}} p_{q} b i_{q}\right)\left(\prod_{l \in \mathcal{P}} p_{l} a^{s_{l}} i_{l}\right): t(A) \rightarrow A
$$

coincide, because their restrictions to any direct summand $A_{l}$ of $t(A)$ are equal (respectively) to the right-hand and the left-hand sides of the relation

$$
\begin{aligned}
i p_{l} b i_{l} p_{l} a^{s_{l}} i_{l}=\left(i p_{l} b\right)\left(i_{l} p_{l} a^{s_{l}} i_{l}\right) & =\left(i p_{l} b\right)\left(a^{s_{l}} i_{l}\right) \\
& =\left(i p_{l} a\right)\left(b^{s_{l}} i_{l}\right)=\left(i p_{l} a\right)\left(i_{l} p_{l} b^{s_{l}} i_{l}\right)=i p_{l} a^{s_{l}} i_{l} p_{l} b i_{l}
\end{aligned}
$$

(to obtain this formula, we have used Lemma 3 and the permutability of the elements $a$ and $b$ ). Now we easily prove the desired statement:

$$
\begin{aligned}
b c & =b i\left(\prod_{l \in \mathcal{P}} p_{l} a^{s_{l}} i_{l}\right) a^{\prime}=i\left(\prod_{q \in \mathcal{P}} p_{q} b i_{q}\right)\left(\prod_{l \in \mathcal{P}} p_{l} a^{s_{l}} i_{l}\right) a^{\prime} \\
& =i\left(\prod_{l \in \mathcal{P}} p_{l} a^{s_{l}} i_{l}\right)\left(\prod_{q \in \mathcal{P}} p_{q} b i_{q}\right) a^{\prime}=i\left(\prod_{l \in \mathcal{P}} p_{l} a^{s_{l}} i_{l}\right) a^{\prime} b=c b .
\end{aligned}
$$

Lemma 7. Let $a^{\prime}$ be a homomorphism from $A$ to $t(A)$, and let $a=i a^{\prime}$. Next, let $b \in E$ be an endomorphism of $A$ such that $b a=a b$. Then there exists an idempotent endomorphism $d$ of $A$ with the following properties: $d$ factors through the periodic part $t(A)$ of $A ; d$ commutes with $b$; and $a(1-d)$ is a weakly nilpotent endomorphism of $A$. 
Proof. It is well known that for any element $x$ of an arbitrary finite semigroup there is an integer $n>0$ such that the element $x^{n}$ is the unity of the semigroup generated by $x$. Since the endomorphism ring of the object $A_{l}$ is finite and $p_{l} a i_{l}$ belongs to this ring, we can find an integer $s_{l}>0$ such that $p_{l} a^{s_{l}+1} i_{l}=\left(p_{l} a i_{l}\right)^{s_{l}+1}$ is the unity of the semigroup of endomorphisms of $A_{l}$ generated by $p_{l} a i_{l}$, and it is easy to check that the $\left(s_{l}+1\right)$ st power of the element $p_{l} a i_{l}-p_{l} a^{s_{l}+2} i_{l}$ is equal to 0 . Set $c=i\left(\prod_{l \in \mathcal{P}} p_{l} a^{s_{l}} i_{l}\right) a^{\prime}$; obviously, the endomorphism $c \in E$ defined by the above formula factors through $t(A)$, and Lemma 6 shows that it commutes with $b$. We put $d=c^{2}$. Since $i a^{\prime} i=a i=i \prod_{l \in \mathcal{P}} p_{l} a i_{l}$, so that $a^{\prime} i=i \prod_{l \in \mathcal{P}} p_{l} a i_{l}$, we easily obtain

$$
d=i\left(\prod_{l \in \mathcal{P}} p_{l} a^{2 s_{l}+1} i_{l}\right) a^{\prime}, \quad d^{2}=i\left(\prod_{l \in \mathcal{P}} p_{l} a^{4 s_{l}+3} i_{l}\right) a^{\prime},
$$

which means that $d$ is an idempotent endomorphism of $A$. In a similar way we check that $a(1-d)$ is a weakly nilpotent endomorphism of $A$.

\section{§6. Category $\bar{M}$}

In this section we shall substantially reduce the study of the objects of the category $M$ to the case where the endomorphism rings of all objects have no additive torsion. For this, we introduce a new category $\bar{M}$.

Let $A, B$ be objects of $M$. Obviously, the set of all homomorphisms from $A$ to $B$ that factor through the torsion $t(B)$ is a subgroup of $\operatorname{Hom}_{M}(A, B)$. Now we define the category $\bar{M}$. Its objects are exactly the objects of the category $M$, but for the homomorphism group $\operatorname{Hom}_{\bar{M}}(A, B)$ we take the factor group of $\operatorname{Hom}_{M}(A, B)$ over the subgroup of homomorphisms that factor through the torsion 2 The following statements show that the category $\bar{M}$ is a good approximation to the category $M$.

Theorem 2. Two objects $A, B$ of $M$ become isomorphic in $\bar{M}$ if and only if there are direct decompositions $A=A_{1} \oplus A_{2}, B=B_{1} \oplus B_{2}$ in $M$ such that $A_{1}$ and $A_{2}$ are isomorphic in $M$, and $B_{1}$ and $B_{2}$ are periodic objects.

Corollary. An object $A$ of $M$ is isomorphic to the zero object in $\bar{M}$ if and only if $A$ is a periodic object.

Theorem 3. If an object $A$ of $M$ is isomorphic in $\bar{M}$ to the direct sum of objects $B$ and $C$ of $M$, then there exists a direct decomposition $A=B^{\prime} \oplus C^{\prime}$ in the category $M$ such that $B^{\prime}$ is isomorphic to $B$ and $C^{\prime}$ is isomorphic to $C$ in $\bar{M}$.

Corollary. An object $A$ of $M$ is indecomposable in $\bar{M}$ if and only if it is almost indecomposable in $M$.

Theorem 4. For any objects $A, B$ of $M$, the group $\operatorname{Hom}_{\bar{M}}(A, B)$ is a torsion free Abelian group of finite rank.

Proof. These theorems were proved in [3] in the case of the category of mixed Abelian groups. The proofs of Theorems 2 and 3 were based on a lemma similar to our Lemma 7 . The proofs in [3] can be modified to serve the general case that we consider here, with the only change: we must refer to Lemma 7 instead of its particular case used in $[3$. As to Theorem 4, it follows directly from Lemma 5 and the assumption $(* * *)$, which says that $\operatorname{Hom}_{M}(A, B)$ is a group without nonzero divisible elements.

\footnotetext{
${ }^{2}$ There was an inaccuracy in the Russian original of the paper: the latter subgroup was called the subgroup of almost zero morphisms, though the notion of an almost zero morphism was never introduced.
} 


\section{REFERENCES}

[1] D. K. Faddeev, An introduction to the multiplicative theory of modules of integral representations, Trudy Mat. Inst. Steklov. 80 (1965), 145-182; English transl. in Proc. Steklov Inst. Math. No. 80 (1968). MR0206048 (34:5873)

[2] A. V. Yakovlev, Torsion-free Abelian groups of finite rank and their direct decompositions, Zap. Nauchn. Sem. S.-Peterburg. Otdel. Mat. Inst. Steklov. (LOMI) 175 (1989), 135-153; English transl., J. Soviet Math. 57 (1991), no. 6, 3524-3533. MR.1047246 (91e:20038)

[3] A. V. Yakovlev and N'Famara Kamara, Mixed Abelian groups of finite rank and their direct decompositions, Vestnik S.-Peterburg. Univ. Mat. Mekh. Astronom. 1993, vyp. 2, 57-61; English transl., Vestnik St. Petersburg Univ. Math. 26 (1993), no. 2, 50-53. MR1370233 (96k:20112)

Department of Mathematics and Mechanics, St. Petersburg State University, Universitetskil Prospekt 28, Stary ̌ Peterhof, St. Petersburg 198504, Russia

E-mail address: yakovlev.anatoly@gmail.com

Received 10/OCT/2007

Translated by THE AUTHOR 